\title{
Linear representation of energy-dependent Hamiltonians
}

\author{
Miloslav Znojil \\ Ústav jaderné fyziky AV ČR, 25068 Řež, Czech Republic \\ e-mail: znojil@ujf.cas.cz
}

\begin{abstract}
Quantum mechanics abounds in models with Hamiltonian operators which are energy-dependent. A linearization of the underlying Schrödinger equation with $H=H(E)$ is proposed here via an introduction of a doublet of separate energyindependent representatives $K$ and $L$ of the respective right and left action of $H(E)$. Both these new operators are non-Hermitian so that our formalism admits a natural extension to non-Hermitian initial $H(E)$ s. Its applicability may range from pragmatic phenomenology and variational calculations (where all the subspace-projected effective operators depend on energy by construction) up to perturbation theory and quasi-exact constructions.
\end{abstract}

PACS 02.30.Tb 03.65Ca 03.65.Db 03.65.Ge 


\section{Introduction}

In non-relativistic quantum mechanics the role of time is exceptional as it remains, up to a few really exotic models [1], unquantized. This makes the current Schrödinger equation $i \hbar \partial_{t} \psi(t)=H \psi(t)$ easily integrated in all the time-independent cases where we get $\psi(t)=\exp \left[i\left(t-t_{0}\right) H\right] \psi\left(t_{0}\right)$. This means that we just have to solve the time-independent Schrödinger equation

$$
H\left|\Psi^{(n)}\right\rangle=E^{(n)}\left|\Psi^{(n)}\right\rangle
$$

and evaluate $\psi(t)$ using the spectral representation of the Hamiltonian operator,

$$
H=\sum_{n}\left|\Psi^{(n)}\right\rangle E^{(n)}\left\langle\Psi^{(n)}\right|
$$

The picture becomes less transparent in all the time-dependent regimes where we have to deal, e.g., with the time-dependent boundary conditions [2] and where the definition of $H=H(t)$ becomes transformed and transferred to $H=H(E)$ in an appropriate Fourier-transformation modification of eq. (1),

$$
H\left(E^{(n)}\right)\left|\Psi^{(n)}\right\rangle=E^{(n)}\left|\Psi^{(n)}\right\rangle .
$$

In our present short note we intend to make a few comments concerning the latter "non-linear" Schrödinger equation.

\section{Motivation}

\subsection{An emergence of the energy-dependence in physics}

The energy-dependent version (3) of the current bound-state Schrödinger equation (1) is far from being an exotic problem without any practical relevance. Almost an opposite statement is true, at least in principle. Indeed, whenever we contemplate a "real" system and construct its manifestly energy-independent "realistic" Hamiltonian $H_{R}$ with due care, we must keep trace of many "possibly relevant" degrees of freedom. In the next step we usually decide, on some

more or less intuitive grounds, that the "really relevant" part of $H_{R}$ might be just its projection $H_{(M S)}=P H_{R} P$ on a certain "model" Hilbert subspace. 
Having finished such a procedure (which is quite common, say, in nuclear physics [3]) one is obliged to compare the spectra $\left\{E_{R}\right\}$ (of $H_{R}$ ) and $\left\{E_{(M S)}\right\}$ (of $H_{(M S)}$ ) and to verify, in this way, the quality and/or reliability of our initial intuition. A formal tool is provided by perturbation theory [4] in terms of which we may arrive at a more quantitative verification of our hypotheses by the almost elementary replacement of the "exact" equation $H_{R}\left|\Psi_{R}\right\rangle=E_{R}\left|\Psi_{R}\right\rangle$ by its two projections $P$ and $Q=1-P$,

$$
\begin{aligned}
& P\left(H_{R}-E_{R}\right) P\left|\Psi_{R}\right\rangle+P\left(H_{R}-E_{R}\right) Q\left|\Psi_{R}\right\rangle=0, \\
& Q\left(H_{R}-E_{R}\right) P\left|\Psi_{R}\right\rangle+Q\left(H_{R}-E_{R}\right) Q\left|\Psi_{R}\right\rangle=0 .
\end{aligned}
$$

Eliminating the irrelevant projection $Q\left|\Psi_{R}\right\rangle$ we easily arrive at the strictly equivalent reduced eigenvalue problem $H_{(r e d)}\left(E_{R}\right)|\varphi\rangle=E_{R}|\varphi\rangle$ of the form (3) with

$$
H_{(\text {red })}(E)=\left\{H_{R}+H_{R} Q\left[\frac{Q}{Q\left(H_{R}-E\right) Q}\right] Q H_{R}\right\} .
$$

Using such a construction of $H_{(r e d)}=H_{(r e d)}(E)$, all the energy errors vanish and we have just the identical energy spectra $\left\{E_{R}\right\} \equiv\left\{E_{(r e d)}\right\}$, in spite of the arbitrary reduction $\left|\Psi_{R}\right\rangle \rightarrow P\left|\Psi_{R}\right\rangle \equiv|\varphi\rangle$ of the Hilbert space itself.

\subsection{Energy-dependent models in computations}

The role of the above exact formula (4) in practical computations is indirect because the energy-dependent operator difference between the exact $H_{R}$ and reduced $H_{(r e d)}\left(E_{R}\right)$ is, undoubtedly, hardly obtainable in closed form. This means that the unique and exact parameter-dependence of $H_{(r e d)}(z)$ is too complicated. In practical computations it invariably requires a perturbative, variational or at least empirical or trial-and-error simplification $H_{(r e d)}(z) \rightarrow H(z)$. At all the relevant real values of $z$ this re-opens the possibility of employing the spectral decomposition,

$$
H(z)=\sum_{n}\left|\Psi^{(n)}(z)\right\rangle E^{(n)}(z)\left\langle\Psi^{(n)}(z)\right|, \quad z \in\left\{E_{R}^{(m)}\right\}_{m=0}^{\infty} .
$$

We see that the work with the energy-dependent Hamiltonians may be both feasible and useful [5]. One only has to keep in mind that it still requires 
comparatively lengthy calculations because at any pre-selected superscript we must guarantee that the value of $z$ satisfies the self-consistency condition

$$
z_{\text {phys }}=E^{(n)}\left(z_{\text {phys }}\right)
$$

This means that the algorithm of construction of bound states generated by any energy-dependent Hamiltonian $H(E)$ remains non-linear, with all the unpleasant mathematical consequences including the possible non-existence or redundancy of the real (and even complex) solutions of eq. (6) at every $n$.

\section{Examples of applicability}

\subsection{Energy scale and its subdomains}

\subsubsection{Thresholds in phenomenological physics}

In the majority of phenomenological models $H_{p h}$, one does not specify any upper limit $E_{\max }$ of their applicability. Thus, atomic and nuclear systems are very often described by non-relativistic Schrödinger equations with interactions reduced to a local two-body phenomenological potential $V=V\left(x_{i}-x_{j}\right)[6]$. Of course, the credibility of such a model becomes very low at the higher energies so that we tacitly have to assume the existence of a complete, two-component description

$$
\begin{array}{ll}
H=H_{\text {lowen. }} & E \leq E_{\text {crit }} . \\
H=H_{\text {highen. }} & E>E_{\text {crit }} .
\end{array}
$$

where the precise localization of the onset of the new physics (expected to occur somewhere near the "threshold" energy $E_{\text {crit. }} \approx 300 \mathrm{MeV}$ in nuclear physics)

is rarely considered really important because, for years, the detailed structure of $H_{\text {high en. }}$ has been believed to be prohibitively complicated. Still, due to the steady increase of our computing power, the high-energy parts of eq. (7) and of its step-function energy-dependence generalizations

$$
H=H_{a, b} \text { for } E \in\left(E_{a}, E_{b}\right)
$$

might acquire the status of a tractable problem quite soon $[5,7]$. 


\subsubsection{Smoothly $E$-dependent Hamiltonians}

One of the best known theoretical sources of differences between the respective low- and high-energy Hamiltonians are relativistic phenomena (e.g., the decays and the emergence of various new degrees of freedom) and corrections (say, in the form of a weakly energy-dependent effective mass in nuclear [8] and particle [9] physics). In such a setting, the building of a model should start in the relativistic kinematical regime and proceed towards the lower energies by a systematic simplification of $H_{\text {highen. }}$. One usually works with a well defined energy-dependence in $H_{\text {highen. }}(E)$ which is smooth. A wealth of explicit examples of this type fits in the scheme of eq. (8) and occurs in numerous applications [10].

Naturally, the study of the models $H(E)$ with a smooth energy dependence attracts attention also by its methodic aspects. To grasp their flavor, the reader is recommended to play with the harmonic-oscillator example of ref. [7],

$$
-\frac{\hbar^{2}}{2 m\left(E^{(n)}\right)} \frac{\mathrm{d}^{2}}{\mathrm{dx}^{2}} \Psi^{(n)}(x)+g x^{2} \Psi^{(n)}(x)=E^{(n)} \Psi^{(n)}(x),
$$

an extremely easy solution of which illustrates many paradoxes exhibited by much more complicated realistic models.

\subsection{Energy-dependence as a formal freedom}

\subsubsection{Non-linearity and non-orthogonality}

The key constraint (6) may be satisfied at a large as well as empty set of real roots at a given $H(E)$ and $n$,

$$
z_{\text {phys }}=E^{(n, 1)}, E^{(n, 2)}, \ldots, E^{(n, m(n))} .
$$

In a compactified notation using multi-indices $\alpha=(n, j)$ we shall abbreviate $E^{(n, j)}=E_{\alpha}$ and denote $\left|\Psi^{(n)}\left(z_{\text {phys }}\right)\right\rangle=\left|\phi_{\alpha}\right\rangle$ in order to identify characteristic difficulties which arise in connection with the energy dependence in $H=H(E)$.

First of all, we have to emphasize that the standard orthogonality relations between the separate bound states become lost. Even though the norm $\left\|\phi_{\alpha}\right\|$ 
of each particular eigenstate may easily be fixed through evaluation of the selfoverlaps $\left\langle\phi_{\alpha} \mid \phi_{\alpha}\right\rangle$, it is necessary to evaluate also all the off-diagonal overlaps

$$
\left\langle\phi_{\alpha} \mid \phi_{\beta}\right\rangle=R_{\alpha, \beta}
$$

which need not non-vanish in general. Secondly, whenever we have to deal with an energy-dependent model where the energies remain discrete, we may alter the denotation of $H=H\left(E_{\alpha}\right)=H\left(E^{(n, j)}\right)=H^{(n)}\left(E^{(n, j)}\right)$. In particular, the latter convention may prove suitable whenever the partial energy-independence property

$$
H^{(n)}\left(E^{(n, j)}\right)=H^{(n)}\left(E^{(n, k)}\right), \quad j, k=1,2, \ldots, m(n)
$$

is encountered as a generalization of the step-shaped energy dependence (8).

\subsubsection{Quasi-exact solvability}

We shall see below that one of the most important technical assumptions of the feasibility of the work with any $H=H(E)$ is the feasibility of the evaluation of the overlap matrix $R(10)$. This is only trivial in the completely solvable and fully energy-independent limit of eq. (9) (with $m(E)=$ const and with the safely diagonal $R_{\alpha, \beta}$ ) where the evaluation of the sequence of the non-vanishing overlaps may be reduced to the formula

$$
R_{\alpha, \alpha}^{\text {(harm.oscil. })} \sim \sum_{n=0}^{N(\alpha)} c_{n} \int_{0}^{\infty} e^{-x^{2}} x^{\text {const }+2 n} d x
$$

which evaluates to a sum of $\Gamma$-functions in both the Hermitian [11] and nonHermitian [12] cases. Difficulties with the determination of $R$ perceivably increase whenever one tries to move to a more realistic model. Vice versa, the requirement of the preservation of a manifestly non-numerical form of the overlaps $R$ leads directly to one of the eligible definitions of the concept of the so called quasi-exact (QE) solvability [13]. For an explicit illustration of this statement, let us pick up one of the most popular QE models, viz., the sextic QE anharmonic-oscillator generalization of eq. (9) which, in the units $\hbar^{2}=2 m(E)=C=1$, reads

$$
\left[-\frac{\mathrm{d}^{2}}{\mathrm{dr}^{2}}+A\left(E_{\alpha}\right) r^{2}+B r^{4}+C r^{6}\right] \Psi_{\alpha}(r)=E_{\alpha} \Psi_{\alpha}(r) \text {. }
$$


This QE model was discovered by Singh et al as early as in the late seventies [14] but its analytic continuation to a non-Hermitian regime appeared only very recently [15]. In both these cases we may use our above composite-index notation with $\alpha=(N, j)$ and $j=1,2, \ldots, m(N)$ and emphasize that the bound-state solutions of eq. (13) remain "quasi-exact" (i.e., elementary and proportional to polynomials) if and only if we keep the integer $N$ fixed. We must select an appropriate specific "spring constant" $A\left(E_{\alpha}\right)=A\left(E^{(N, j)}\right) \equiv A_{N}$ which is different for the different $N=1,2, \ldots$. In this manner one introduces a certain "minimally complicated" energy dependence in the Hamiltonian (13). At the same time, all the sums and integrals which define the diagonal as well as non-diagonal overlaps $R_{\alpha, \beta}$ remain very similar to eq. (12) and may be easily shown to degenerate to finite sums of incomplete $\Gamma$-functions.

\section{Hermitian Hamiltonians $H(E)$}

\subsection{Bi-orthogonality and completeness}

In eq. (10), a nontrivial assumption is needed as a guarantee that the matrix $R$ has an inverse (for the time being, let us also keep in mind that it is, by construction and/or assumption, Hermitian). Only then, the formal decomposition of the identity projector becomes available as a double sum over the suitable (sub)set $A$ of the multi-indices,

$$
\hat{I}=\sum_{\alpha, \beta \in A}\left|\phi_{\alpha}\right\rangle\left(R^{-1}\right)_{\alpha, \beta}\left\langle\phi_{\beta}\right|
$$

Even if we knew the set $A$ and proved the convergence, the practical value of such a formula would remain significantly lowered by the non-diagonality of the infinite-dimensional matrix $R$. The sufficiently precise evaluation of this matrix and of its inverse $R^{-1}$ is needed. As we already mentioned (cf. also [16]), all this requires a sufficiently elementary form of the basis states $\left|\phi_{\alpha}\right\rangle$.

Equation (14) acquires the standard linear-algebraic meaning of a "completeness" relation for our (non-orthogonal) basis $\left\{\left|\phi_{\alpha}\right\rangle\right\}_{\alpha \in A}$. After we abbre- 
viate

$$
\left\langle\left\langle\phi_{\alpha}\right|=\sum_{\beta \in A}\left(R^{-1}\right)_{\alpha, \beta}\left\langle\phi_{\beta}\right|\right.
$$

a new basis emerges which, by construction, obeys the Kronecker-delta-overlap rule

$$
\left\langle\left\langle\phi_{\alpha} \mid \phi_{\beta}\right\rangle=\left\langle\phi_{\alpha} \mid \phi_{\beta}\right\rangle\right\rangle=\delta_{\alpha, \beta}, \quad \alpha, \beta \in A
$$

and is called, on this ground, bi-orthogonal and bi-orthonormal [17].

\subsection{Two alternative quasi-Hermitian representants}

Practical use of the bi-orthogonally generalized basis exhibits a lot of parallels with its ordinary orthogonal predecessor. First of all, it enables us to reinterpret the double series (14) as a single-index expansion employing the two different types of vectors,

$$
\hat{I}=\sum_{\alpha \in A}\left|\phi_{\alpha}\right\rangle\left\langle\left\langle\phi_{\alpha}\left|=\sum_{\alpha \in A}\right| \phi_{\alpha}\right\rangle\right\rangle\left\langle\phi_{\alpha}\right| .
$$

It is easy to verify that the new operator defined by its generalized spectral representation

$$
K=\sum_{\alpha, \beta \in A}\left|\phi_{\alpha}\right\rangle E_{\alpha}\left(R^{-1}\right)_{\alpha, \beta}\left\langle\phi_{\beta}\right|
$$

or, in an abbreviated notation,

$$
K=\sum_{\alpha \in A}\left|\phi_{\alpha}\right\rangle E_{\alpha}\left\langle\left\langle\phi_{\alpha}\right|\right.
$$

may be re-interpreted as an operator which shares with $H(E)$ its action to the right,

$$
K\left|\phi_{\alpha}\right\rangle=E_{\alpha}\left|\phi_{\alpha}\right\rangle, \quad \alpha \in A \text {. }
$$

By construction, the new operator $K$ is energy-independent (i.e., no nonlinearity is encountered). The latter property is counterbalanced by non-Hermiticity $K \neq K^{\dagger}$ which means that the action of $K$ to the left is much more complicated. Nevertheless, we may complement the linear, non-Hermitian operator $K$ by another operator, viz., by

$$
L=\sum_{\alpha, \beta \in A}\left|\phi_{\alpha}\right\rangle\left(R^{-1}\right)_{\alpha, \beta} E_{\beta}\left\langle\phi_{\beta}\right| .
$$


This means that

$$
\left\langle\phi_{\alpha} \mid L=E_{\alpha}\left\langle\phi_{\alpha}\left|, \quad L=\sum_{\beta \in A}\right| \phi_{\beta}\right\rangle\right\rangle E_{\beta}\left\langle\phi_{\beta}\right|
$$

so that the second non-Hermitian linear operator $L$ shares with $H(E)$ its action to the left. In this sense, the single nonlinear operator $H(E)$ may be understood as represented by the linear doublet $(K, L)$.

One has to notice that $K=L^{\dagger}$ and $L=K^{\dagger}$ are mutually inter-related,

$$
\xi L=K \xi=\sum_{\alpha \in A}\left|\phi_{\alpha}\right\rangle E_{\alpha}\left\langle\phi_{\alpha}\left|, \quad \xi=\sum_{\alpha \in A}\right| \phi_{\alpha}\right\rangle\left\langle\phi_{\alpha}\right|=\xi^{\dagger}
$$

or, alternatively,

$$
\left.L \xi^{-1}=\xi^{-1} K=\sum_{\alpha \in A}\left|\phi_{\alpha}\right\rangle\right\rangle E_{\alpha}\left\langle\left\langle\phi_{\alpha}\left|, \quad \xi^{-1}=\sum_{\alpha \in A}\right| \phi_{\alpha}\right\rangle\right\rangle\left\langle\left\langle\phi_{\alpha}\right| .\right.
$$

As long as we have $\xi>0$, the terminology of the review paper [18] may be used implying that both our mutually conjugate Hamilton-like operators $K$ and $L$ are quasi-Hermitian. For this reason, each of them admits a consistent quantum-mechanical interpretation (readers are recommended to check ref. [18] for more details).

\section{Non-Hermitian Hamiltonians $H(E)$}

Recently, several groups of authors [19] - [24] tried to weaken the traditional Hermiticity $H=H^{\dagger}$ [tacitly also assumed, up to now, in formulae (2) and (5) above]. The standard pattern of such a generalization (which, for the sake of simplicity, keeps the energies real) lies in the assumption that the bra and ket vectors in the similar spectral expansions are not the mere Hermitian conjugates of each other. This resembles the above-described relation between the vectors $\left|\phi_{\alpha}\right\rangle$ and $\left.\left|\phi_{\alpha}\right\rangle\right\rangle$ which we also assumed to remain significantly different. Indeed, many rules of our preceding section 4 will find their parallels also in the forthcoming text where we shall admit that $H(E) \neq H^{\dagger}(E)$.

\subsection{Quasi-Hermitian input Hamiltonians $H(E)$}

Let us keep the superscripted kets $\left|\Psi^{(n)}\right\rangle$ of eq. (2) unchanged and introduce another, independent infinite series of their subscripted partners $\left|\Psi_{(n)}\right\rangle \neq\left|\Psi^{(n)}\right\rangle$. 
In the light of the current textbooks [17] the usual orthogonality (and normalization) assumption $\left\langle\Psi^{(m)} \mid \Psi^{(n)}\right\rangle=\delta_{m, n}$ finds its most natural generalization in the so called bi-orthogonality (or rather bi-orthonormality) relations between these two sets,

$$
\left\langle\Psi_{(m)} \mid \Psi^{(n)}\right\rangle=\delta_{m, n}, \quad m, n=1,2, \ldots
$$

In parallel, the usual completeness relations for a basis in our Hilbert space $\mathcal{H}$ must be replaced by the following innovated, bi-orthogonal-basis-related formula

$$
\hat{I}=\sum_{n}\left|\Psi^{(n)}\right\rangle\left\langle\Psi_{(n)}\right|
$$

This enables us to replace the spectral decomposition (2) of Hermitian $H=H^{\dagger}$ by its non-Hermitian bi-orthogonal-basis analogue or generalization,

$$
H=\sum_{n}\left|\Psi^{(n)}\right\rangle E^{(n)}\left\langle\Psi_{(n)}\right| \neq H^{\dagger}
$$

Parallels are preserved with eqs. (16) and/or (15),

$$
\begin{gathered}
H^{\dagger} \eta=\eta H=\sum_{n}\left|\Psi_{(n)}\right\rangle E^{(n)}\left\langle\left\langle\Psi_{(n)}\left|, \quad \eta=\sum_{n}\right| \Psi_{(n)}\right\rangle\left\langle\Psi_{(n)}\right|=\eta^{\dagger},\right. \\
H \eta^{-1}=\eta^{-1} H^{\dagger}=\sum_{n}\left|\Psi^{(n)}\right\rangle E^{(n)}\left\langle\left\langle\Psi^{(n)}\left|, \quad \eta^{-1}=\sum_{n}\right| \Psi^{(n)}\right\rangle\left\langle\Psi^{(n)}\right| .\right.
\end{gathered}
$$

We may call again all our generalized Hamiltonians (18) quasi-Hermitian [18] since we are working with the manifestly regular and positive $\eta>0$ in the latter two relations.

\subsection{Innovated pair of the representants $K$ and $L$}

Once we keep in mind the quasi-Hermiticity symmetries (19) and (20), we may introduce an error-checking convention under which we only consider the formulae where all the kets are upper-indexed while all the bras are lowerindexed. Under this convention the extension of the results of section 4 to all the quasi-Hermitian $H(E) \neq H^{\dagger}(E)$ is straightforward. Firstly, in the way inspired by eq. (5) we have to define

$$
H(z)=\sum_{n}\left|\Psi^{(n)}(z)\right\rangle E^{(n)}(z)\left\langle\Psi_{(n)}(z)\right|
$$


and re-abbreviate

$$
\left|\varphi^{\alpha}\right\rangle=\left|\Psi^{(n)}\left(z_{\text {phys }}\right)\right\rangle, \quad\left\langle\varphi_{\alpha}\right|=\left\langle\Psi_{(n)}\left(z_{\text {phys }}\right)\right|
$$

where the rising or lowering of the index means a transition to another, entirely different vector. Assuming the knowledge of all the overlaps

$$
\left\langle\varphi_{\alpha} \mid \varphi^{\beta}\right\rangle=R_{\alpha}^{\beta}
$$

(notice an inessential change in our matrix indexing), we have to emphasize that they do not form a Hermitian matrix anymore. Still, its assumed invertibility suffices for us to write down the unit projector

$$
\left.\hat{I}=\sum_{\alpha, \beta \in A}\left|\varphi^{\alpha}\right\rangle\left(R^{-1}\right)_{\alpha}^{\beta}\left\langle\varphi_{\beta}\left|=\sum_{\beta \in A}\right| \varphi^{\beta}\right\rangle\right\rangle\left\langle\varphi_{\beta}\left|=\sum_{\alpha \in A}\right| \varphi^{\alpha}\right\rangle\left\langle\left\langle\varphi_{\alpha}\right|\right.
$$

where we abbreviated

$$
\left.\sum_{\alpha \in A}\left|\varphi^{\alpha}\right\rangle\left(R^{-1}\right)_{\alpha}^{\beta} \equiv\left|\varphi^{\beta}\right\rangle\right\rangle, \quad \sum_{\beta \in A}\left(R^{-1}\right)_{\alpha}^{\beta}\left\langle\varphi_{\beta}\right| \equiv\left\langle\left\langle\varphi_{\alpha}\right|\right.
$$

This means that

$$
\left\langle\left\langle\varphi_{\alpha} \mid \varphi^{\beta}\right\rangle=\left\langle\varphi_{\alpha} \mid \varphi^{\beta}\right\rangle\right\rangle=\delta_{\alpha}^{\beta}, \quad \alpha, \beta \in A
$$

and that

$$
\hat{I}=\sum_{\alpha \in A}\left|\varphi^{\alpha}\right\rangle\left\langle\left\langle\varphi_{\alpha}\left|=\sum_{\alpha \in A}\right| \varphi^{\alpha}\right\rangle\right\rangle\left\langle\varphi_{\alpha}\right| .
$$

It is now trivial to define the generalized operators $K$ and $L$ which share with the quasi-Hermitian $H(E)$ its action to the right and left, respectively,

$$
\begin{gathered}
K=\sum_{\alpha \in A}\left|\varphi^{\alpha}\right\rangle E_{\alpha}\left\langle\left\langle\varphi_{\alpha}|\quad K| \varphi^{\beta}\right\rangle=E_{\beta} \mid \varphi^{\beta}\right\rangle, \\
\left\langle\varphi_{\alpha} \mid L=E_{\alpha}\left\langle\varphi_{\alpha}\left|, \quad L=\sum_{\beta \in A}\right| \varphi^{\beta}\right\rangle\right\rangle E_{\beta}\left\langle\varphi_{\beta}\right| .
\end{gathered}
$$

This completes our construction. 


\section{Summary: Separation of the left and right action of the energy-dependent Hamiltoni- ans in Hilbert space}

When we compare the results of sections 4 and 5 we may feel surprised by the smoothness of the transition to the non-Hermitian $H(E)$. In fact, the only perceivable consequence of the loss of the Hermiticity of $H(E)$ lies in the emergence of the independent operator of the inverted metric which is defined in terms of the new, upper-indexed bras and kets. Formally, the breakdown $K \neq L^{\dagger}$ and $L \neq K^{\dagger}$ of the conjugation symmetry only implies that the quasi-Hermiticity rules (19) and (20) must be replaced by their appropriate modifications

$$
\left.K^{\dagger} \mu=\mu K=\sum_{\alpha \in A}\left|\varphi_{\alpha}\right\rangle\right\rangle E_{\alpha}\left\langle\left\langle\varphi_{\alpha}\left|, \quad \mu=\sum_{\alpha \in A}\right| \varphi_{\alpha}\right\rangle\right\rangle\left\langle\left\langle\varphi_{\alpha}\right|=\mu^{\dagger}\right.
$$

and

$$
\nu L=L^{\dagger} \nu=\sum_{\alpha \in A}\left|\varphi_{\alpha}\right\rangle E_{\alpha}\left\langle\varphi_{\alpha}\left|, \quad \nu=\sum_{\alpha \in A}\right| \varphi_{\alpha}\right\rangle\left\langle\varphi_{\alpha}\right|=\nu^{\dagger}
$$

where we must keep in mind that

$$
\left.\mu^{-1}=\sum_{\alpha \in A}\left|\varphi^{\alpha}\right\rangle\left\langle\varphi^{\alpha}\left|, \quad \nu^{-1}=\sum_{\alpha \in A}\right| \varphi^{\alpha}\right\rangle\right\rangle\left\langle\left\langle\varphi^{\alpha}\right| .\right.
$$

This means that we in fact violated the "error-correcting" convention of section

5 and started writing all the "redundant" Hermitian-conjugate formulae in their explicit form. Such a step facilitates the most concise formulation of our present message stating that the original operator $H(E)$ and its conjugate $H^{\dagger}(E)$ are in fact subtly different in both the Hermitian and non-Hermitian cases. In the other words, even after the present "linearization" of their action in the standard, self-dual Hilbert space, none of their two energy-independent representatives $K$ and $L$ is redundant.

\section{Acknowledgements}

Work supported by GA AS CR, grant Nr. 1048302 and by the AS CR projects K1048102, K1010104 and AV0Z1048901. 


\section{References}

[1] E. P. Wigner, Rev. Mod. Phys. 29 (1957) 255;

J. Hilgevoord, Am. J. Phys. 70 (2002) 301, with further references.

[2] P. Šeba, Phys. Rev. A 41 (1990) 2306;

J. Dittrich and P. Duclos, J.Phys. A 35 (2002) 8213, with further references.

[3] H. Feshbach, Ann. Phys. (NY) 5 (1958) 357;

P. Navrátil, H. B. Geyer and T. T. S. Kuo, Phys. Lett. B 315 (1993) 1, with further references.

[4] K. Kumar, Perturbation Theory and the Nuclear Many Body Problem, North Holland, Amsterdam, 1962;

F. M. Fernández, Introduction to Perturbation Theory in Quantum Mechanics, CRC Press, Boca Raton, 2001.

[5] J. Formánek, R. J. Lombard and J. Mareš, Czech. J. Phys. 54 (2004) 289;

H. Bíla, Pseudo-Hermitian Hamiltonians in Quantum Theory (diploma thesis, in Czech, unpublished), Charles University, Prague, April 2004, chapter 2 .

[6] M. Znojil, Czech. J. Phys. B 30 (1980) 488;

P. Bydžovský and M. Sotona, Czech. J. Phys. 48 (1998) 903, with references.

[7] M. Znojil, H. Bíla and V. Jakubskyý, Energy-dependent Hamiltonians and their pseudo-Hermitian interpretation, LANL arXiv quant-ph/0312148, unpublished.

[8] W. Weise, Nucl. Phys. A 278 (1977) 402;

B. D. Serot and J. D. Walecka, Adv. Nucl. Phys. 16 (1986) 1;

E. Friedman, A. Gal and J. Mareš, Nucl. Phys. A 625 (1997) 272, with references. 
[9] P. Lichard, Phys. Rev. D 55 (1997) 5385 and 60 (1999) 053007.

[10] H. A. Bethe H A and E. F. Salpeter E E 1957 Quantum theory of Oneand Two-Electron Systems, Handbuch der Physik, Band XXXV, Atome I, Springer Verlag, Berlin, 1957;

M. Hirata, F. Lenz and K. Yazaki, Ann. Phys. (NY) 108 (1977) 116;

E. D. Cooper, B. K. Jennings and J. Mareš, Nucl. Phys. A 580 (1994) 419.

[11] A. Messiah, Quantum Mechanics II, North Holland, Amsterdam, 1961.

[12] M. Znojil, Phys. Lett. A 259 (1999) 220;

B. Bagchi, C. Quesne and M. Znojil, Mod. Phys. Lett. A 16 (2001) 2047;

M. Znojil, Rendic. Circ. Mat. Palermo Ser. II, Suppl. 72 (2004) 211;

S. Weigert, Czech. J. Phys. 54 (2004) 147.

[13] A. G. Ushveridze, Quasi-exactly Solvable Models in Quantum Mechanics, IOPP, Bristol, 1994.

[14] V. Singh, S. N. Biswas and K. Datta, Phys. Rev. D 18 (1978) 1901.

[15] F. Cannata, M. Ioffe, R. Roychoudhury and P. Roy, Phys. Lett. A 281 (2001) 305.

[16] M. Znojil, New types of solvability in PT symmetric quantum theory, in Proceedings of Workshop on Superintegrability in Classical and Quantum Systems (September 16-21, 2002, Montreal, Canada), to appear.

[17] T. Ya. Azizov and I. S. Iokhvidov, Linear Operators in Spaces with Indefinite Metric, Wiley, Chichester, 1989.

[18] F. G. Scholtz, H. B. Geyer and F. J. W. Hahne, Ann. Phys. (NY) 213 (1992) 74 .

[19] P. A. M. Dirac, Proc. Roy. Soc. London A 180 (1942) 1;

W. Pauli, Rev. Mod. Phys. 15 (1943) 175;

H. Feshbach and F. Villars, Rev. Mod. Phys. 30 (1958) 24; 
C. M. Bender and K. A. Milton, Phys. Rev. D 55 (1997) R3255;

A. Ramirez and B. Mielnik, Rev. Mex. Fis. 49S2 (2003) 130, giving a concise history of the subject.

[20] M. Robnik and M. V. Berry, J. Phys. A: Math. Gen. 19 (1986) 669;

N. Hatano and D. R. Nelson, Phys. Rev. Lett. 77 (1996) 570;

W. D. Heiss and H. L. Harney, Eur. Phys. J. D 17 (2001) 149.

[21] C. M. Bender and S. Boettcher, Phys. Rev. Lett. 80 (1998) 4243;

P. Dorey, C. Dunning and R. Tateo, J. Phys. A: Math. Gen. 34 (2001) 5679 ;

C. M. Bender, D. C. Brody and H. F. Jones, Phys. Rev. Lett. 89 (2002) 270401;

C. M. Bender, P. N. Meisinger and and Q. Wang, J. Phys. A: Math. Gen. 36 (2003) 1973.

[22] E. Caliceti, S. Graffi and M. Maioli, Commun. Math. Phys. 75 (1980) 51;

V. Buslaev and V. Grecchi, J. Phys. A: Math. Gen. 26 (1993) 5541;

F. Cannata, G. Junker and J. Trost, Phys. Lett. A 246 (1998) 219;

F. M. Fernández, R. Guardiola, J. Ros and M. Znojil, J. Phys. A: Math. Gen. 31 (1998) 10105;

G. Lévai, F. Cannata and A. Ventura, Phys. Lett. A 300 (2002) 271 and J. Phys. A: Math. Gen. 35 (2002) 5041.

[23] A. Mostafazadeh, J. Math. Phys. 43 (2002) 205, 2841 and 3944 and Czech. J. Phys. 53 (2003) 1079 and Phys. Lett. A 320 (2004) 375.

[24] Collection of all 20 papers in Nr. 1 of Czech. J. Phys. 54 (2004), pp. 1 bis 156. 